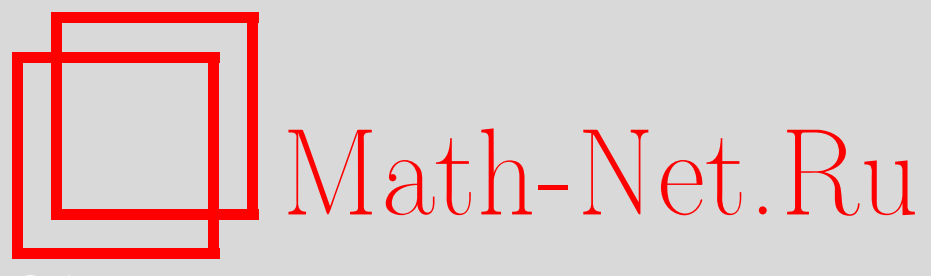

А. Л. Таламбуца, Достижимость минимального показателя экспоненциального роста для некоторых фуксовых групп, Матем. заметки, 2010, том 88, выпуск 1, 152-156

DOI: https://doi.org/10.4213/mzm8800

Использование Общероссийского математического портала Math-Net.Ru подразумевает, что вы прочитали и согласны с пользовательским соглашением http://www.mathnet.ru/rus/agreement

Параметры загрузки:

IP : 54.147 .182 .235

26 апреля 2023 г., 15:00:36

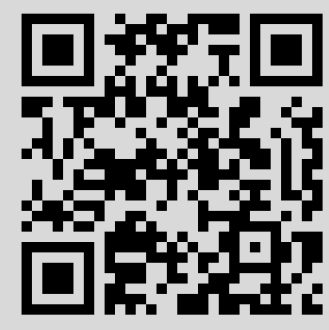




\section{Достижимость минимального показателя экспоненциального роста для некоторых фуксовых групп}

\section{А. Л. Таламбуца}

Пусть группа $G$ порождена конечным множеством элементов $S$. Функиией роста $F_{G, S}(m)$ группы $G$ относительно $S$ называется количество элементов группы $G$, выразимых групповыми словами длины не более $m$ в алфавите $S$. Из очевидного неравенства $F_{G, S}(k+m) \leqslant F_{G, S}(k) F_{G, S}(m)$ следует (см. [1; предложение VI.C.56]), что существует предел

$$
\lambda_{G, S}=\lim _{m \rightarrow \infty}\left(F_{G, S}(m)\right)^{1 / m} .
$$

Этот предел называется показателем экспоненииального роста группы $G$ относительно множества порождающих $S$. Так как далее будут рассматриваться только такие показатели роста, то слово "экспоненциальный” для краткости будем опускать. Минимальным показателем роста группы $G$ называется точная нижняя грань $\lambda_{G}=\inf _{S} \lambda_{G, S}$ по всем конечным множествам порождающих $S$ группы $G$.

В книге [1; VII.19.Е] был поставлен вопрос: для каких классов групп экспоненциального роста минимальный показатель роста достигается на некотором множестве порождающих? Там же было приведено несложное рассуждение, показывающее, что в свободной группе ранга $k \geqslant 2$ минимальный показатель роста достигается и равен $2 k-1$.

По-видимому достижимость минимального показателя роста была доказана лишь для указанного выше примера свободных групп и свободных произведений $\mathbb{Z}_{p} * \mathbb{F}_{k}$ циклической группы простого порядка $p$ на свободную группу ранга $k$ (см. [2]). Также были найдены примеры групп, для которых минимальный показатель роста не достигается ни на каком множестве порождающих (см. [3]) и даже может быть равен 1 (см. [4]).

В работе будет доказано, что минимальный показатель роста достигается для групп

$$
H_{g, n}=\left\langle s_{1}, t_{1}, \ldots, s_{g}, t_{g} \mid\left(\left[s_{1}, t_{1}\right] \cdots\left[s_{g}, t_{g}\right]\right)^{n}=1\right\rangle \quad \text { при } \quad n>1 .
$$

Эти группы естественным образом связаны с фундаментальными группами двумерных ориентируемых поверхностей, а также хорошо известны как фуксовы группы сигнатуры $(g ; n)$. Действительно, добавив один порождающий элемент $z=\left(\left[s_{1}, t_{1}\right] \cdots\left[s_{g}, t_{g}\right]\right)^{-1}$, указанное задание группы $H_{g, n}$ можно переписать как следующее каноническое задание фуксовой группы с одним эллиптическим элементом $z$ порядка $n$ :

$$
\left\langle s_{1}, t_{1}, \ldots, s_{g}, t_{g}, z \mid z\left[s_{1}, t_{1}\right] \cdots\left[s_{g}, t_{g}\right]=1, z^{n}=1\right\rangle .
$$

ЛЕмма. В любом конечном множестве, порождающем группу $G=H_{g, n}$, есть подмножество из $2 g$ элементов, порождающее в $G$ подгруппу, изоморфную $\mathbb{F}_{2 g}$ или $H_{g, n}$.

Доказательство. Пусть $K=[G, G]$ и $\varphi$ - естественный эпиморфизм $G \rightarrow G / K$. Ясно, что факторгруппа $G^{a b}=G / K$ изоморфна свободной абелевой группе ранга $2 g$. Далее будет удобно рассматривать группу $G^{a b}$ как модуль размерности $2 g$ над кольцом целых чисел и понимать элементы группы еще и как векторы этого модуля.

Пусть группа $G$ порождена некоторым множеством $S=\left\{a_{1}, \ldots, a_{m}\right\}$; тогда $G^{a b}$ порождается системой векторов $\varphi(S)=\left\{\varphi\left(a_{1}\right), \ldots, \varphi\left(a_{m}\right)\right\}$. Так как $\operatorname{dim}\left(G^{a b}\right)=2 g$, в системе $\varphi(S)$ существует линейно независимая подсистема $\left\{\varphi\left(a_{i_{1}}\right), \ldots, \varphi\left(a_{i_{2 g}}\right)\right\}$. Докажем, что ее прообраз, множество $T=\left\{a_{i_{1}}, \ldots, a_{i_{2 g}}\right\}$, удовлетворяет условию леммы.

Работа выполнена при поддержке Российского фонда фундаментальных исследований и программы "Ведущие научные школы" (грант № НШ-65648.2010.1). 
Обозначим через $\mathrm{Gp}(T)$ подгруппу, порожденную множеством $T$ в группе $G$. Через $\operatorname{rank}(K)$, как обычно, обозначается ранг группы $K$, т.е. наименьшее возможное число порождающих для нее. В силу линейной независимости системы $\varphi(T)$ выполнены равенства

$$
\operatorname{rank}(\varphi(\operatorname{Gp}(T)))=\operatorname{rank}(\mathrm{Gp}(T))=2 g .
$$

Будем использовать классический результат (см. [5; предложение III.7.4]) о том, что всякая подгруппа фуксовой группы бесконечного индекса является свободным произведением циклических групп, а подгруппа конечного индекса сама является фуксовой.

Рассмотрим вначале случай $[G: G p(T)]=\infty$. Тогда $\operatorname{Gp}(T)$ имеет задание вида

$$
\left\langle t_{1}, \ldots, t_{k}, z_{1}, \ldots, z_{r} \mid z_{i}^{c_{i}}=1, i=1, \ldots, r\right\rangle,
$$

ранг которого равен $k+r$ по следствию из теоремы Грушко-Неймана (см. [5; с. 246]). Таким образом, в силу (1) выполнено равенство $2 g=k+r$.

Так как $G^{a b}$ - группа без кручения, $\varphi\left(z_{i}\right)=1$, и поэтому ранг $\varphi(\operatorname{Gp}(T))$ не превосходит $k$. Отсюда сразу следует $k \geqslant 2 g$, что вместе с неравенством $2 g=k+r$ сразу влечет $k=2 g$ и $r=0$. При этом, очевидно, задание (2) представляет собой задание свободной группы $\mathbb{F}_{2 g}$, и лемма в этом случае доказана.

Пусть теперь $\operatorname{Gp}(T)$ есть подгруппа конечного индекса в $G$, т.е. $[G: \operatorname{Gp}(T)]$ есть некоторое натуральное число. Как уже было сказано, $\operatorname{Gp}(T)$ есть фуксова группа и, значит, она обладает заданием вида

$$
\left\langle x_{1}, y_{1}, \ldots, x_{h}, y_{h}, z_{1}, \ldots, z_{r} \mid\left[x_{1}, y_{1}\right] \cdots\left[x_{h}, y_{h}\right] z_{1} z_{2} \cdots z_{r}=1, z_{i}^{c_{i}}=1, i=1, \ldots, r\right\rangle .
$$

Вначале опять заметим, что при отображении $\varphi$ элементы $z_{i}$ переходят в единицу и, значит, при абелизации $G$ подгруппа $\operatorname{Gp}(T)$ переходит в некоторую свободную абелеву группу ранга не более $2 h$, откуда в силу равенства (1) получаем $h \geqslant g$.

Из теоремы 3 работы [6] следует, что ранг фуксовой группы вида (3) равен $2 h+r-1$ при $r>0$ и равен $2 h$ при $r=0$. Так как $\operatorname{rank}(\operatorname{Gp}(T))=2 g$, в обоих случаях отсюда следует, что полученное выше неравенство $h \geqslant g$ влечет точное равенство $h=g$. При этом для количества элементов конечного порядка в задании (3) остаются только два варианта: $r=0$ или $r=1$.

Покажем, что на самом деле случай $r=0$ невозможен. Действительно, в этом случае формула Римана-Гурвица для подгруппы $\operatorname{Gp}(T)$ в группе $G$ имеет следующий вид:

$$
2 g-2=[G: \operatorname{Gp}(T)] \cdot\left(2 g-2-\left(1-\frac{1}{n}\right)\right) .
$$

При $g=1$ левая часть равенства обращается в 0 , а правая - нет, получаем противоречие. При $g>1$ величины $A=2 g-2$ и $B=2 g-2-(1-1 / n)$ обе больше единицы, но разница $A-B$ мала, и поэтому $A$ и $B$ не могут быть кратны друг другу с целым множителем $[G: \operatorname{Gp}(T)] \neq 1$. Действительно, $A=2 g-2 \geqslant 2$, при этом $B=2 g-2-(1-1 / n)>1$, а разница $A-B=1-1 / n<1$.

Перейдем теперь к разбору случая $r=1$. Для $g>1$ формула Римана-Гурвица

$$
2 g-2-\left(1-\frac{1}{c_{1}}\right)=[G: \operatorname{Gp}(T)] \cdot\left(2 g-2-\left(1-\frac{1}{n}\right)\right)
$$

может быть выполнена только в тривиальном случае $[G: \operatorname{Gp}(T)]=1$, опять же в силу того, что числа $2 g-2-\left(1-1 / c_{1}\right)$ и $2 g-2-(1-1 / n)$ мало отличаются друг от друга.

При $g=1$ формула Римана-Гурвица записывается как

$$
1-\frac{1}{c_{1}}=[G: \operatorname{Gp}(T)] \cdot\left(1-\frac{1}{n}\right) .
$$


Так как $1-1 / c_{1}$ и $(1-1 / n)$ - числа, лежащие в полуинтервале $[1 / 2,1)$, то равенство $(5)$ возможно лишь в случае $c_{1}=n$. Получаем, что $\operatorname{Gp}(T) \cong H_{1, n}$, и лемма в этом случае также верна.

Теорема 1. Минимальный показатель роста группы $G=H_{g, n}$ nри $n>1$ достигается на некотором множестве порождающих, состоящем из $2 g$ элементов.

ДокАЗАтЕЛьСтво. Это рассуждение с незначительными изменениями повторяет доказательство основной теоремы из [2].

Пусть $\mu_{G}=\inf _{S} \lambda_{G, S}$, где $S$ пробегает только те множества порождающих группы $G$, которые состоят из $2 g$ элементов. Покажем, что для любого множества $S$ порождающих группы $G$ выполнено неравенство

$$
\lambda_{G, S} \geqslant \mu_{G}
$$

Воспользовавшись леммой, выберем в множестве $S$ подмножество $T$ из $2 g$ элементов, такое, что $\operatorname{Gp}(T) \cong H_{g, n}$ или $\mathrm{Gp}(T) \cong \mathbb{F}_{2 g}$. В обоих случаях выполнено неравенство

$$
\lambda_{\mathrm{Gp}(T), T} \geqslant \mu_{G} .
$$

Действительно, в случае $\mathrm{Gp}(T) \cong H_{g, n}$ оно является следствием определения $\mu_{G}$, а в случае $\operatorname{Gp}(T) \cong \mathbb{F}_{2 g}$ оно вытекает из оценки $\lambda_{\mathrm{Gp}(T), T}=4 g-1 \geqslant \mu_{G}$. Объединив $(7)$ с очевидным неравенством $\lambda_{G, S} \geqslant \lambda_{\mathrm{Gp}(T), T}$, получим требуемое неравенство (6).

Очевидно, из (6) следует, что $\lambda_{G}=\mu_{G}$, а это значит, что для доказательства теоремы осталось показать, что минимальный показатель роста группы $G$ достигается в классе множеств порождающих, состоящих из $2 g$ элементов.

Известно (см. [7; теорема 5]), что всякое множество $S$ порождающих группы $H_{g, n}$, состоящее из $2 g$ элементов, может быть приведено цепочкой нильсеновских преобразований к стандартному множеству порождающих $S_{0}=\left\{s_{1}, t_{1}, \ldots, s_{g}, t_{g}\right\}$. Отсюда следует, что в порождающих $S=\left\{a_{1}, \ldots, a_{2 g}\right\}$ группа $H_{g, n}$ имеет задание

$$
\left\langle a_{1}, \ldots, a_{2 g} \mid R_{S}\left(a_{1}, \ldots, a_{2 g}\right)^{n}=1\right\rangle .
$$

Для группы с заданием вида (8) в работе [2] была доказана следующая нижняя оценка на показатель экспоненциального роста:

$$
\lambda_{G, S} \geqslant(4 g-1)^{1-\left(1+\left\lceil\log _{3}|R|\right\rceil\right) /\lfloor(n-1)|R| / 3\rfloor} .
$$

Так как в стандартном задании группы $G$ используется $2 g$ порождающих и есть нетривиальное соотношение, из жесткости роста свободных групп (см. [3; теорема 1.1]) следует, что для некоторого $\varepsilon>0$ выполнено равенство $\lambda_{G, S_{0}}=(4 g-1)^{1-\varepsilon}$. Пусть тогда $l_{0}$ - такое натуральное число, что

$$
\varepsilon>\frac{1+\left\lceil\log _{3} l_{0}\right\rceil}{\left\lfloor(n-1) l_{0} / 3\right\rfloor}
$$

оно существует, так как правая часть неравенства монотонно убывает к 0 при $l_{0} \rightarrow \infty$. Тогда получаем, что если в задании (8) длина слова $R_{S}$ больше $l_{0}$, то выполнено строгое неравенство

$$
\lambda_{G, S_{R}}>\lambda_{G, S_{0}} .
$$

Существует лишь конечное число различных заданий $\mathscr{P}$ группы $G$ с $2 g$ порождающими и единственным соотношением, длина которого не превосходит $l_{0}$, и поэтому на множестве $\mathscr{P}$ минимум показателей роста достигается, а из неравенства (11) следует, что этот минимум будет больше, чем у остальных заданий группы $G$.

В случае $g=1$ теорему 1 можно уточнить следующим образом.

Теорема 2. Минимальный показатель роста группы $G=H_{1, n}$ nри $n>1$ достигается на любом двухэлементном множестве порождающих и равен $1 / z_{0}$, где $z_{0}$ единственный корень многочлена $P_{n}(z)=z^{2 n+1}-3 z^{2 n}+3 z-1$, лежащий в интервале $(1 / 3,1 / 2)$. 
ДокАЗАтЕльство. В силу теоремы 1 минимальный показатель роста группы $H_{1, n}$ достигается на некотором множестве порождающих, состоящем из двух элементов.

Пусть $S=\{u, v\}$ - некоторое такое множество порождающих группы $G$; тогда, как уже отмечалось, $S$ может быть переведено в $S_{0}$ цепочкой нильсеновских преобразований. Каждое такое преобразование переводит коммутатор $W=[x, y]$ в слово вида $K W^{\varepsilon} K^{-1}$, где $\varepsilon \in\{-1,1\}$. Значит, в порождающих $S$ группа $G$ имеет задание $G=\left\langle u, v \mid[u, v]^{n}=1\right\rangle$. Очевидно, что тогда $F_{G, S}(m)=F_{G, S_{0}}(m)$ для всех $m$, и поэтому $\lambda_{G, S}=\lambda_{G, S_{0}}$.

Из основной теоремы работы [8] легко можно вычислить производящую функцию для сферической функции роста группы $H_{g, n}$ относительно стандартного множества порождающих. В случае $g=1$ она принимает следующий вид:

$$
\Delta_{n}(z)=\frac{1+2 z+2 z^{2}+\cdots+2 z^{2 n-2}+2 z^{2 n-1}+z^{2 n}}{1-2 z-\cdots-2 z^{2 n-1}+z^{2 n}} .
$$

Производящая функция $\Sigma_{n}(z)$ обычной (шаровой) функции роста $f_{G, S_{0}}(m)$ получается из $\Delta_{n}(z)$ делением на $(1-z)$, и поэтому может быть записана как

$$
\Sigma_{n}(z)=\frac{1+2 z+2 z^{2}+\cdots+2 z^{2 n-2}+2 z^{2 n-1}+z^{2 n}}{1-3 z+3 z^{2 n}-z^{2 n+1}} .
$$

Тогда показатель роста $\lambda_{G, S_{0}}$ есть $1 / \beta$, где $\beta$ - радиус круга сходимости ряда Маклорена, задаваемого функцией (12). В нашем случае, когда функция $\Sigma_{n}(z)$ рациональная, этот радиус совпадает с минимальным абсолютным значением всех полюсов функции.

Напомним, что многочлен из знаменателя дроби (12) отличается от многочлена $P_{n}(z)$ только знаком, и поэтому имеет те же корни. Легко проверяется, что для любого $n>$ 1 на концах интервала $(1 / 3,1 / 2)$ многочлен $P_{n}(z)$ меняет знак, и поэтому у него есть некоторый действительный корень $z_{0}$, лежащий в этом интервале.

Рассмотрим на комплексной плоскости круг радиуса $1 / 2$ с центром в точке 0. Для этого круга и монома первой степени многочлена $P_{n}(z)$ применима теорема Руше о количестве комплексных корней многочлена (см. [9]). Действительно, при $n>1$ выполнено неравенство

$$
3 \cdot \frac{1}{2}>1+3 \cdot\left(\frac{1}{2}\right)^{2 n}+\left(\frac{1}{2}\right)^{2 n+1} .
$$

Это означает, что внутри круга у многочлена $P_{n}(z)$ существует ровно один корень, а именно, найденный выше действительный корень $z_{0}$.

Заметим, что многочлен из числителя дроби (12) может быть представлен в виде

$$
(z+1)\left(z^{2 n-1}+\cdots+z+1\right)=\frac{(z+1)\left(z^{2 n}-1\right)}{z-1}
$$

откуда очевидно, что все его комплексные корни лежат на единичной окружности, и среди них нет $z_{0}$. Таким образом, точка $z_{0}$ является минимальным по модулю полюсом функции $\Sigma_{n}(z)$, что завершает доказательство теоремы.

Приведем вычисленные приближенно значения минимальных показателей роста групп $H_{1, n}$ для $n=2, \ldots, 6$ :

$$
\begin{gathered}
\lambda_{H_{1,2}} \approx 2,890054, \quad \lambda_{H_{1,3}} \approx 2,988825, \quad \lambda_{H_{1,4}} \approx 2,998777, \\
\lambda_{H_{1,5}} \approx 2,999864, \quad \lambda_{H_{1,6}} \approx 2,999985 .
\end{gathered}
$$

Имеет место предельное равенство $\lim _{n \rightarrow \infty} \lambda_{H_{1, n}}=3$, которое следует из теоремы 2 и нижней оценки показателя роста (9), примененной к множеству порождающих $S_{0}$. 


\section{СПИСОК ЦИТИРОВАННОЙ ЛИТЕРАТУРЫ}

[1] P. de la Harpe, Topics in Geometric Group Theory, Chicago Lectures in Math., Univ. Chicago Press, Chicago, IL, 2000. [2] А.Л. Таламбуца, Матем. заметки, 78:4 (2005), 614-618. [3] A. Sambusetti, Ann. Sci. École Norm. Sup. (4), 35:4 (2002), 477-488. [4] J. S. Wilson, Invent. Math., 155:2 (2004), 287-303. [5] Р. Линдон, П. Шупп, Комбинаторная теория груnп, Мир, М., 1980. [6] N. Peczynski, G. Rosenberger, H. Zieschang, Invent. Math., 29:2 (1975), 161-180. [7] G. Rosenberger, Monatsh. Math., 84:1 (1977), 55-68. [8] M. Stall, Some Group Presentations with Rational Growth, http:// www.faculty.jacobs-university.de/mstoll/papers/ratgrow.dvi. [9] А. И. Маркушевич, Теория аналитических функций. т. 1: Начала теории, Наука, М., 1967.

\section{А. Л. Таламбуца}

Поступило

Математический институт им. В. А. Стеклова РАН

18.02.2010

E-mail: altal@mi.ras.ru 